Meta

Journal des traducteurs

Translators' Journal

\title{
Déterritorialisation et reterritorialisation : l'importation de contre-modèles dans le champ de la traduction
}

\section{Laurent Lamy}

Volume 60, numéro 2, août 2015

$60^{\mathrm{e}}$ anniversaire. Les horizons de la traduction : retour vers le futur $60^{\text {th }}$ Anniversary. Translation's Horizons: Back to the Future

60mo aniversario. Los horizontes de la traducción: regreso al futuro

URI : https://id.erudit.org/iderudit/1032903ar

DOI : https://doi.org/10.7202/1032903ar

Aller au sommaire du numéro

Éditeur(s)

Les Presses de l’Université de Montréal

ISSN

0026-0452 (imprimé)

1492-1421 (numérique)

Découvrir la revue

Citer ce document

Lamy, L. (2015). Déterritorialisation et reterritorialisation : l'importation de contre-modèles dans le champ de la traduction. Meta, 60(2), 352-352.

https://doi.org/10.7202/1032903ar 


\title{
Déterritorialisation et reterritorialisation : l'importation de contre-modèles dans le champ de la traduction
}

\author{
LAURENT LAMY \\ Université de Montréal, Montréal, Canada \\ laurent.lamy@umontreal.ca
}

Cette présentation offre un aperçu de la migration et du nomadisme des concepts pouvant contribuer à un élargissement des horizons du champ de la réflexion traductologique. Notre propos s'inscrit dans l'axe multipolaire de la transversalité et de la perméabilité transfrontalière des savoirs et des pratiques. Nous examinerons plus spécialement le modèle du rhizome mis de l'avant par Gilles Deleuze et Félix Guattari dans Mille plateaux. Ils le décrivent comme un processus de déterritorialisation et de reterritorialisation à ramifications multiples. Nous nous attacherons plus précisément aux trois principes de la prolifération rhizomatique, par contraste avec la contiguilté fortement hiérarchisée du modèle arborescent hérité d'Aristote, soit la connexion, l'hétérogénéité et la multiplicité: «Un chaînon sémiotique est comme un tubercule agglomérant des actes très divers, linguistiques, mais aussi perceptifs, mimiques, gestuels, cogitatifs: il n'y a pas de langue en soi, ni d'universalité du langage, mais un concours de dialectes, de patois, d'argots, de langues spéciales. Il n'y a pas de locuteur-auditeur idéal, pas plus que de communauté linguistique homogène» (Deleuze et Guattari 1980: 14). Cette composante est ouverte et non saturable. Diverses multiplicités hétérogènes cohabitent, se parasitent et s'interconnectent, car elles «se définissent par le dehors: par la ligne abstraite, ligne de fuite ou de déterritorialisation suivant laquelle elles changent de nature en se connectant avec d'autres» (Deleuze et Guattari 1980: 15). Nous prenons ensuite appui sur l'image du rhizome que forment l'orchidée et la guêpe telle que décrite par ces auteurs, en référence aux mouvements de déterritorialisation et aux procès de reterritorialisation, comme modèle alternatif pour décrire un processus de traduction comme «capture de code», "plus-value de code» connectant, déterritorialisant et reterritorialisant des devenirs hétérogènes. Nous pouvons en tirer une analogie très féconde pour décrire le processus réel qui s'engage dans la traduction. Finalement, nous démontrerons que cette connectivité multilatérale présente une nette affinité avec une conception de la traduction élaborée déjà par Walter Benjamin, dans son essai de 1916 Über Sprache überhaupt und über die Sprache des Menschen, à savoir que «la traduction est le transfert d'une langue dans l'autre à travers un continuum de métamorphoses. La traduction traverse des continuums de métamorphose, non pas des zones abstraites d'équivalence et de ressemblance» (Benjamin 1991: 150; notre traduction).

Formé en philologie et en philosophie, poète publié, traducteur et théoricien de la traduction, Laurent Lamy est chargé de cours et chercheur associé au Département de linguistique et de traduction de l'Université de Montréal. Parmi ses nombreuses publications théoriques sur la traduction, notons «Vortex linguarum: de la traduction comme remix: du post- au trans-» dans Mutatis Mutandis (2013), «Steiner et Meschonnic: pour en finir avec la fable de l'indicible et le spectre de l'objection préjudicielle» dans Belas Infiéis (2013) et «Conjecture/conjoncture/ ouverture: une vue non déterministe de la traduction (Popper, Kuhn et Feyerabend "revisités") » dans Meta (2014). 